Die Zweistimmenkonstruktion wird unter dem Gesichtspunkt erörtert, welche taktischen und strategischen Möglichkeiten ein Stimmensplitting bietet. Leider reizt der Autor die Thematik dabei nicht voll aus. Einen Reformvorschlag unterbreitet Behnke beim Zweistimmensystem nicht, wohl deswegen, weil er keine großen Möglichkeiten zur Manipula-tion sieht. Die Wähler empfinden das jedoch vielfach anders und neigen zum Stimmensplitting auch deshalb, weil sie glauben, dadurch besonders geschickt zu handeln. Gegen eine Abschaffung des Zweistimmensystems, wie sie unter anderem der Rezensent fordert, sprächen "gewichtige demokratietheoretische Gründe“ (S. 202), die Behnke nicht eigens benennt. Der Ertrag der Ausführungen zum Zweistimmensystem fällt suboptimal aus.

Sollte die Union nur dank möglicher Überhangmandate gemeinsam mit den Liberalen bei der Bundestagswahl 2009 eine Mandatsmehrheit erlangen, könnte ein Legitimationsproblem eintreten. Behnke nimmt zu Recht kein Blatt vor den Mund, spricht gar, etwas überzogen, von einer „demokratietheoretischen Katastrophe“ (S. 235). „So bleibt also nur zu hoffen, dass sich die Einsicht, dass eine Reform des Wahlgesetzes nicht nur überfällig, sondern auch ein Gebot politischer und demokratischer Klugheit ist, in nächster Zeit durchsetzen wird“ (S. 235). Diese Hoffnung geht für die nächste Wahlperiode nach 2009 auf, denn das Bundesverfassungsgericht hat in seinem Urteil vom 3. Juli 2008 dem passiv gebliebenen Gesetzgeber zu Recht aufgetragen, das Wahlverfahren bis zum 30. Juni 2011 so zu ändern, dass ein ,inverser Erfolgswert“ unmöglich gemacht wird. Der Rezensent seinerseits hofft: Mögen Überhangmandate das Ergebnis nicht in das Gegenteil verkehren.

Eckhard Jesse

\title{
Bundeswahl- und Parteiengesetz: instruktiv kommentiert
}

Schreiber, Wolfgang: Bundeswahlgesetz (BWahlG). Kommentar, 8. Auflage, Carl Heymanns Verlag, Köln 2009, 1125 Seiten, € 148,-.

Ipsen, Jörn (Hrsg.): Gesetz über die politischen Parteien (ParteienG). Kommentar, Verlag C.H. Beck, München 2008, 588 Seiten, € 88,-.

Wahlrechtsfragen sind oft Machtfragen, jedoch nicht immer, wie das folgende Beispiel zeigt: Der Bundeswahlausschuss, dem der Bundeswahlleiter vorsteht, hat auch bei der diesjährigen Bundestagswahl einige Parteien aus formalen Gründen nicht zugelassen, so die Grauen, die anarchistische APPD und die Freie Union von Gabriele Pauli. Solche Fragen betreffen das Wahlgesetz ebenso wie das Parteiengesetz.

Im Jahr 1975 wurde für die Bundestagswahl 1976 zum ersten Mal das „Handbuch des Wahlrechts zum Deutschen Bundestag“ auf den Markt gebracht - unter der Ägide von Wolfgang Schreiber, des damaligen Wahlrechtsreferenten im Bundesministerium des Innern. Der Kommentar zum Bundeswahlrecht von Karl-Heinz Seifert wurde danach nicht mehr publiziert. ${ }^{1}$ Vor jeder folgenden Bundestagswahl - abgesehen von der vorgezogenen 2005

1 Vgl. Karl-Heinz Seifert, Bundeswahlrecht. Kommentar, 3. Auflage, München 1976. 
- erschien eine Neuauflage. Nun liegt die achte vor, diesmal unter dem griffigeren Titel „Kommentar zum Bundeswahlgesetz“. „Die Reduzierung des Titels hat verlegerische Gründe“ (S. VII). Der „Schreiber“ ist gewohnt aktuell. Alle Änderungen, so die Novellierung des Gesetzes vom 17. März 2008, sind berücksichtigt worden. Zu den wichtigsten gehören das neue Berechnungsverfahren (von Hare / Niemeyer zu Sainte-Laguë / Schepers), die zeitlich unbefristete Gewährung des Wahlrechts für Auslandsdeutsche sowie die Neuordnung des Listennachfolgeverfahrens. Auch die Novellierungen zur Bundeswahlordnung fehlen nicht. Ferner sind neuere Entscheidungen des Bundesverfassungsgerichts eingehend kommentiert, ebenso Reformüberlegungen (etwa zum breit und kontrovers diskutierten Wahlrecht von Geburt an).

Schreiber, Honorarprofessor an der Martin-Luther-Universität Halle-Wittenberg, urteilt durchweg kompetent. Das zeigt sich etwa an den „Erläuterungen zum Bundeswahlgesetz“ (S. 25 bis 85), die der Kommentierung der einzelnen Paragraphen vorgeschaltet sind. Im Anhang (S. 835 bis 1019) findet sich unter anderem der Text der Bundeswahlordnung, ehe ein ausführliches Literaturverzeichnis folgt. Das Ziel, „für alle in Rechtsprechung, Rechtswissenschaft sowie in der Wahl-, Parlaments- und Parteienpraxis auf Bundes- und Länderebene an Wahlrechtsfragen Interessierten und beruflich mit dieser Rechtsmaterie befassten und an der Vorbereitung und Durchführung von Wahlen Beteiligten eine - auf wissenschaftlicher Basis erstellte und zugleich praxisorientierte - aktuelle und detaillierte Informationsquelle und Orientierungshilfe zu allen Fragen des Bundestagswahlrechts zu sein“" (S. 18), ist ohne Wenn und Aber erfüllt. Allerdings: Manchmal hat selbst der mit der Materie Vertraute den Eindruck, den Wald vor lauter Bäumen nicht mehr zu sehen. Weniger wäre mehr gewesen. So ist es nicht einsichtig, wieso manche Fußnoten sich über mehr als zwei Seiten erstrecken. Das hängt damit zusammen, dass der Autor die einschlägige Literatur fortschreibt, nicht immer mit Gespür für deren Relevanz. Und wie sinnvoll etwa ist es, bei einzelnen Titeln auf Besprechungen zu verweisen? Daher darf es nicht verwundern, dass der neueste „Schreiber“ auch der dickste ist.

Der Kommentar zum Parteiengesetz von Jörn Ipsen, Staatsrechtler an der Universität Osnabrück und Präsident des Niedersächsischen Staatsgerichtshofs, zeichnet sich insgesamt eher durch „Schlankheit“ aus. Es hat fast 20 Jahre gedauert, ehe ein Parteiengesetz erlassen wurde - und mehr als 40 Jahre, bevor dieses Gesetz eine systematische Kommentierung erfuhr. Leider umfasst das Vorwort nur eine Seite. Der Zufall will es, dass jetzt, kurz nach dem Ipsen-Kommentar ein weiterer angekündigt ist. ${ }^{2}$

Jörn Ipsen und seine Mitarbeiter Heike Jochum, Thorsten Koch, Frank Saliger, Katrin Stein (bis auf Saliger alle von der Universität Osnabrück) haben einen Kommentar aus einem Guss vorgelegt - von $₫ 1$ bis $₫ 41$. Das Werk besticht durch große Einheitlichkeit. Nach dem Text des jeweiligen Paragraphen folgen Hinweise auf die Entstehungsgeschichte, das Schrifttum und eine Inhaltsübersicht, ehe die Kommentierung an der Reihe ist. Sie ist prägnant, nicht weitschweifig. Ipsen nennt vier Aufgabenbereiche für Parteien beim Namen: die Beteiligung an Wahlen, die Auslese des Führungspersonals, die Artikulations- und Integrationsaufgabe. Etwa die Hälfte des Umfangs nehmen die Ausführungen zur Rechenschaftslegung ein. Dieses heikle Thema hat in der Vergangenheit immer wieder für zum Teil

2 Vgl. Matthias Ross / Sophie-Charlotte Lenski (Hrsg.), Parteiengesetz und Recht der Kandidatenaufstellung, Baden-Baden 2009 (im Erscheinen). 
unerquickliche Zwistigkeiten geführt. Die detaillierten Ausführungen zum Vollzug des Verbots verfassungswidriger Parteien hätten stärker die politische Wirklichkeit berücksichtigen können.

Beide Kommentierungen sind zuverlässig - und überaus gut brauchbar. Die Dichte der Informationen besticht. Wer sucht, wird fündig. Der Nutzen für die Politikwissenschaft wäre bei einer stärkeren Einbeziehung der sozialwissenschaftlichen Erkenntnisse noch größer gewesen. Aber diese Einschränkung tut dem einen wie dem anderen Unterfangen keinen Abbruch.

Eckhard Jesse

\section{Parlamentarische Kontrolle der Exekutive: verfassungsrechtliche Perspektiven}

Schmidt, Jörg: Die demokratische Legitimationsfunktion der parlamentarischen Kontrolle (Schriften zum Öffentlichen Recht, Band 1064), Duncker \& Humblot, Berlin 2007, 485 Seiten, $€ 98,-$.

Teuber, Christian: Parlamentarische Informationsrechte. Eine Untersuchung an den Beispielen des Bundestages und des Landtages Nordrhein-Westfalen (Beiträge zum Parlamentsrecht, Band 63), Duncker \& Humblot, Berlin 2007, 336 Seiten, € 69,80.

Christian Teuber und Jörg Schmidt nehmen sich aus ganz unterschiedlichen verfassungsrechtlichen Perspektiven eines aktuellen Problems an: Wie kann parlamentarische Kontrolle angesichts der Privatisierung einstmals staatlicher Tätigkeit gewährleistet werden? Jörg Schmidt wählt den Weg einer rechtsdogmatischen Auseinandersetzung: Im Zentrum stehen dabei nicht einzelne Instrumente parlamentarischer Kontrolle, sondern die grundlegende Frage, welchen Stellenwert die Kontrolle der Exekutive durch das Parlament in der demokratischen Ordnung des Grundgesetzes hat. Der Autor schließt sich zwar der auch in der politikwissenschaftlichen Forschung üblichen Position an, dass es sich um eine selbständige Parlamentsfunktion handelt, die vorrangig in der Kontrolle der Exekutive besteht. Anders als in der üblichen Betrachtung kann parlamentarische Kontrolle hier aber auch in Gesetzesform wahrgenommen werden, ist nicht vor allem Aufgabe der Opposition, die diese öffentlich wahrnimmt, sondern obliegt wesentlich der Mehrheit. In den Worten von Jörg Schmidt ist parlamentarische Kontrolle „zum einen abstrakte Strukturbedingung der demokratischen Legitimation und zum anderen deren konkrete Ausgestaltung“ (S. 121). Im Unterschied zur Gesetzgebung als positiver Legitimation kommt es bei dieser Form der „negativen Legitimation“ staatlichen Handelns nur auf die Möglichkeit zur Einwirkung und nicht auf tatsächliches Kontrollhandeln an. Aus dieser Konzeption von parlamentarischer Kontrolle als Legitimation ergibt sich, dass es für ihr Vorhandensein eines ununterbrochenen Verantwortungszusammenhangs bedarf, bei dem Entscheidungen zugerechnet werden können und Einflussmöglichkeiten bestehen. Indem der Autor dem Begriff der Verantwortung einen solch zentralen Stellenwert einräumt, betont er die klare Verortung seiner Interpretation der grundgesetzlichen Ordnung in der Tradition repräsentativer Demokratie. 